\title{
Decreased Exhaled Nitric Oxide Levels in Patients with Mitochondrial Disorders
}

\author{
Ricardo A. Mosquera*,, Cheryl L. Samuels², Tomika S. Harris², Aravind Yadav ${ }^{1}$, \\ S. Shahrukh Hashmi ${ }^{3}$, Melissa S. Knight ${ }^{4}$ and Mary Kay Koenig ${ }^{4}$
}

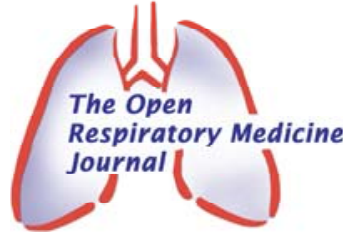

\author{
${ }^{1}$ University of Texas Health Science Center at Houston (UTHealth), School of Medicine, Department of Pediatric \\ Pulmonary, Houston, Texas, USA \\ ${ }^{2}$ UTHealth School of Medicine, Pediatric Comprehensive Care, Houston, Texas, USA \\ ${ }^{3}$ UTHealth School of Medicine, Pediatric Research Center, Department of Pediatrics, Houston, Texas, USA \\ ${ }^{4}$ UTHealth School of Medicine, Department of Pediatric Neurology, Houston, Texas, US.
}

\begin{abstract}
Background: Nitric oxide (NO) deficiency may occur in mitochondrial disorders (MD) and can contribute to the pathogenesis of the disease. It is difficult and invasive to measure systemic nitric oxide. NO is formed in the lungs and can be detected in expired air. Currently, hand-held fractional exhaled nitric oxide (FeNO) measurement devices are available enabling a fast in-office analysis of this non-invasive test. It was postulated that FeNO levels might be reduced in MD.

Methods: Sixteen subjects with definite MD by modified Walker criteria (4 to 30 years of age) and sixteen healthy control subjects of similar age, race and body mass index (BMI) underwent measurement of FeNO in accordance with the American Thoracic Society guidelines.

Results: Sixteen patient-control pairs were recruited. The median FeNO level was 6.5 ppm (IQR: 4-9.5) and 10.5 ppm (IQR: 8-20.5) in the MD and control groups, respectively. In 13 pairs (81\%), the FeNO levels were lower in the MD cases than in the matched controls $(\mathrm{p}=0.021)$. Eleven $(69 \%)$ cases had very low FeNO levels $(\leq 7 \mathrm{ppm})$ compared to only 1 control ( $\mathrm{p}=0.001$ ). All cases with enzymatic deficiencies in complex I had FeNO $\leq 7 \mathrm{ppm}$.

Conclusions: Single-breath exhaled nitric oxide recordings were decreased in patients with MD. This pilot study suggests that hand-held FeNO measurements could be an attractive non-invasive indicator of MD. In addition, measurement of FeNO could be used as a parameter to monitor therapeutic response in this population.
\end{abstract}

Keywords: Mitochondrial disorder, exhaled nitric oxide, FeNO, walker criteria, modified walker criteria, NIOX MINO.

\section{INTRODUCTION}

Following initial descriptions, the term "mitochondrial disorder" (MD) arose to describe primary defects in the mitochondrial electron transport chain [1]. In MD, dysfunctional mitochondria are unable to generate ATP in quantities sufficient to meet the energy needs of high energy tissues and organs. Persons with MD manifest multi-organ symptoms including epilepsy, intellectual disability, skeletal and cardiac myopathy, diabetes mellitus, sensorineural hearing loss, and renal impairments [2,3]. MD can affect persons of any age. Adults typically present with welldefined "mitochondrial syndromes" resulting from mitochondrial DNA mutations that are easily identified. Children with MD are much harder to identify because children are more likely to have nuclear DNA mutations and the "classic" mitochondrial syndromic findings are typically absent [1]. Over the last few decades, diagnostic criteria have

*Address correspondence to this author at the 6431 Fannin Street, MBS 3.226A, Houston, Texas 77030, USA; Tel: 713-500-5650;

E-mail: Ricardo.A.Mosquera@uth.tmc.edu been developed to assist in the recognition and diagnosis of MD. Walker et al. were the first to propose a set of major and minor criteria for the identification of mitochondrial disorders in adults [4]. Bernier et al. revised the criteria of Walker et al. to better suit the pediatric population, proposing a set of modifications to the criteria specific to children. These criteria are now known as the "modified Walker criteria" [5]. As this disease becomes more recognized there is a growing need to improve diagnostic methods for detection of MD and to find parameters allowing monitoring of therapeutic responses in this population.

Nitric oxide (NO) is a biologic messenger produced by mammalian cells serving various regulatory functions including blood flow, platelet adhesion, immunity, and neurotransmission. There is growing evidence that NO deficiency occurs in at least some MD and can play a major role in the pathogenesis of stroke-like episodes, myopathy, diabetes mellitus, and lactic acidosis [3,5-10]. As such, NO measurements in patients with mitochondrial disease could be an important tool for understanding the pathogenesis and stages of the disease, assisting in the diagnosis, and 
monitoring response to treatment. At present it is very difficult to measure authentic NO levels in vivo. Indirect methods have been developed to measure the end products of NO metabolism in biological samples; however, the existing methods are expensive, invasive, and require complex analytical laboratory techniques [11].

NO is produced in the lung by virtually all cellular constituents, including the epithelium, the endothelium, neuroendocrine cells, resident inflammatory cells, and alveolar macrophages [12]. NO production is altered in these cells via various mechanisms such as impaired NO production due to a generalized impairment of endothelial function (endothelial dysfunction), NO shunting into reactive nitrogen species formation, decreased availability of the NO precursors arginine, or increased asymmetric dimethylarginine (ADMA) concentrations [4]. An increase in respiratory NO production can be measured by exhaled NO levels (FeNO).

This level is used as a marker of lung inflammation in patients with asthma, allergic rhinitis, and bronchiectasis to monitor therapy, ie the higher the FeNO level, the higher the inflammation [13]. In contrast, FeNO levels are decreased in several known disease populations including primary pulmonary hypertension, primary cilliary dyskinesia and cystic fibrosis. In primary pulmonary hypertension (PPH) low levels of NO result from pulmonary vasoconstriction and increased vascular resistance [14]. Patients with primary cilliary dyskinesia (PCD) have low FeNO levels due to the decreased activity of the inducible nitric oxide synthase (iNOS) in their airways [15]. Current recommendations include performing a FeNO in order to screen patients before performing the much more invasive biopsy testing [16]. Patients with cystic fibrosis also have low FeNO levels associated with airway obstruction [17]. Currently, handheld FeNO measurement devices are available, enabling a fast in-office analysis of this non-invasive test to help screen or monitor these disease processes.

Decreased levels of the NO precursor arginine have been reported in MD patients but FeNO levels have not been evaluated in this population [3]. We hypothesized that the arginine deficiency seen in these patients might result in reduced levels of FeNO. FeNO levels were measured in subjects with MD and in healthy control subjects with similar age, sex, race, and body mass index.

\section{MATERIALS AND METHODS}

\subsection{Study Subjects}

Sixteen subjects with a definite diagnosis of MD via the modified Walker criteria seen at the University of Texas Mitochondrial Center between September 2012 and January 2013 had exhaled FeNO levels measured. Medical records were reviewed to ascertain if patients were undergoing pharmacologic treatment of MD at the time of inclusion in the study. A study participant was categorized as atopic if a diagnosis of atopy was made by their primary care physician or by an allergist. Patients with Mitochondrial disease and poorly controlled atopy were excluded from the study as atopy can significantly increase level of FeNO. Concurrently exhaled FeNO levels were obtained in sixteen non-atopic, non-smoker healthy control subjects matched by gender, ethnicity and age (+/-1 year if less than 18 years old, +/-4 years if 18 years or older). These healthy control subjects were recruited from patients obtaining regular preventative care at the pediatric clinic located near the MD clinic or were healthy siblings of patients seeking care at UT Physicians. Lack of atopic disease among controls was confirmed by either their primary care physician or from report by the patient or parent. In all subjects, FeNO levels were obtained at baseline without an acute illness. Informed consent was obtained from all study participants or their parents. Due to the lack of significant variability between FeNO readings only one or two readings per subject was obtained [18]. The study was reviewed and approved by the University of Texas Medical School at Houston institutional review board prior to enrolling subjects.

\subsection{Measurements of Nitric Oxide}

Study participants were asked to refrain from eating and drinking for at least 1 hour before measurements. Online single breath oral FeNO measurements were performed using the NIOX MINO® Airway Inflammation Monitor (Aerocrine AB, Solna, Sweden). In a standing position, subjects first exhaled through the mouth down to the residual lung volume and then inhaled through the adapter of the analyzer up to their total lung capacity. Subjects then exhaled 6-10 seconds against an expiratory resistance of 5$20 \mathrm{~cm} \mathrm{H}_{2} \mathrm{O}$ with a constant flow of an average of $50 \mathrm{ml} / \mathrm{s}$ through the mouthpiece of the analyzer monitor. The subject was able to adapt expiratory flow rates by viewing a display showing their flow rate located on a computer screen. The lower limit of detection for the monitor is $5 \mathrm{ppm}$ and readings lower than those are reported as " $<5 \mathrm{ppm}$ ".

\subsection{Data Analysis}

Median and interquartile range (IQR) were calculated for FeNO levels in the MD and control groups. Wilcoxon signed rank tests were used to compare the relative levels of FeNO between each MD-control pair. All subjects with FeNO values below the lower detectable limit (<5 ppm) were assumed to be the same and given an arbitrary value of 4 ppm for the paired analysis. Fisher exact tests were used to compare frequencies between groups. All analyses were performed using Stata (v.10, College Station, TX, USA) and significance was assumed at a two-sided alpha of 0.05.

\section{RESULTS}

Of the sixteen subjects with MD there were nine males and seven females; fifteen were Caucasian and one was Hispanic. Eleven of the subjects were atopic. (Table 1). Most $(\mathrm{n}=13,81 \%)$ were receiving treatment for $\mathrm{MD}$ with supplements (coenzyme Q10, levo-carnitine, L-arginine, folic acid, and vitamin B6) at the time the FeNO level was obtained. All of the MD subjects were non-smokers.

All sixteen age, ethnicity, and gender matched control subjects were without atopic disease. None of the control subjects were taking any regularly prescribed medication. All of the control subjects were non-smokers. Age-adjusted BMI were in the overweight range for two subjects in each of the two groups (Table 1).

FeNO levels were significantly lower in the MD group compared to the controls. The median FeNO level in the MD group was $6.5 \mathrm{ppm}$ (IQR: 4-9.5) compared to $10.5 \mathrm{ppm}$ 
(IQR: 8-20.5) in the control group (Fig. 1). The difference was significant in the matched comparisons where the FeNO levels in the MD case were lower in 13 pairs $(81 \%)$ and higher in only 3 pairs (19\%) compared to their matched control $(\mathrm{p}=0.021)$. Among the pairs where the lower levels were present in the MD cases, the difference in FeNO levels between the pairs ranged from $1 \mathrm{ppm}$ to $17 \mathrm{ppm}$ (median: 6 ppm). Comparatively, in the three pairs where the MD case had a higher FeNO level, the difference in levels was 3 ppm.

Table 1. Demographic Distributions of MD Patients and Healthy Controls (n=16 for Each Group)

\begin{tabular}{|l|c|c|}
\hline Characteristics & MD* Patients & Healthy Controls \\
\hline \hline Age, median (IQR) & $11(7-17)$ & $11(7-16)$ \\
\hline Gender, male, $\mathrm{n}(\%)$ & $9(56 \%)$ & $9(56 \%)$ \\
\hline Race/ethnicity & & \\
\hline non-Hispanic white & $15(94 \%)$ & $15(94 \%)$ \\
\hline Hispanic & $1(6 \%)$ & $1(6 \%)$ \\
\hline BMI & & \\
\hline Overweight, $\mathrm{n}(\%)$ & $2(13 \%)$ & $2(13 \%)$ \\
\hline History of atopy & $11(69 \%)$ & $0(0 \%)$ \\
\hline
\end{tabular}

*MD = Mitochondrial disease.

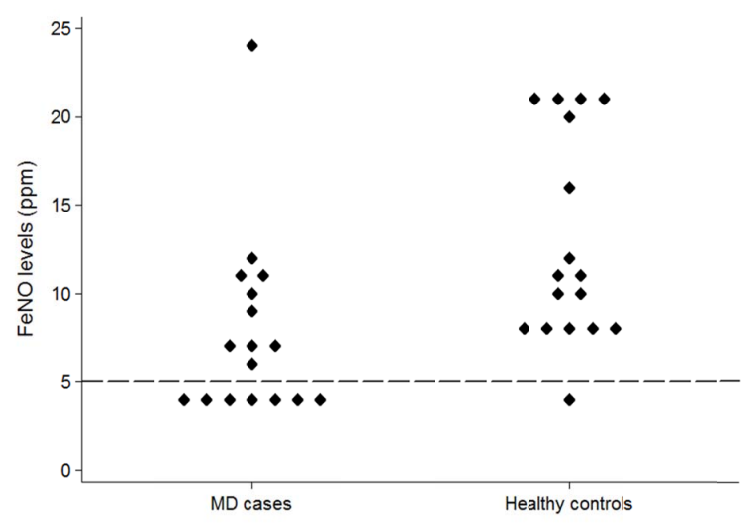

Fig. (1). Distribution of FeNO levels among the MD cases and the age-matched healthy controls. The dashed line represents the lower detection limit of the monitor. Values below 5ppm are reported by the monitor as $<5 \mathrm{ppm}$. For purposes of the graph and analysis, all values $<5 \mathrm{ppm}$ were considered to be the same.

Extremely low levels of FeNO $(\leq 7 \mathrm{ppm})$ were measured in eleven (69\%) MD subjects, including seven who registered below the detection limit of the Niox Mino FeNO device $(<5 \mathrm{ppm})$. This was significantly different than the control subjects where only one subject had an extremely low level of FeNO $(<5 \mathrm{ppm}) \mathrm{p}=0.001)$. Furthermore, among the MD patients, six of the eleven $(55 \%)$ with extremely low levels of FeNO had enzymatic deficiencies in complex I (Table 2). However, this deficiency was not present in any of the MD patients with normal levels of FeNO ( $\mathrm{p}=0.043$ ).
Table 2. FeNO Levels for Each Case/Control Pair and Known Mutation Among Cases

\begin{tabular}{|c|c|c|l|}
\hline \multirow{2}{*}{ Pair ID } & \multicolumn{2}{|c|}{ FeNO Levels (ppm) ** } & \multirow{2}{*}{$\begin{array}{l}\text { Known Mutation } \\
\text { Among Cases * }\end{array}$} \\
\cline { 2 - 4 } & MD Patients * & Healthy Controls & \\
\hline \hline 1 & 11 & 8 & FHx Complex I \\
\hline 2 & $<5$ & 8 & Complex I \\
\hline 3 & $<5$ & 21 & Fiber Type I Predominance \\
\hline 4 & $<5$ & 11 & Complex I \\
\hline 5 & 7 & $<5$ & Complex I \\
\hline 6 & $<5$ & 10 & Complex II \\
\hline 7 & $<5$ & 20 & Complex I+III \\
\hline 8 & 7 & 21 & Complex I andIII \\
\hline 9 & 6 & 8 & Complex I \\
\hline 10 & 11 & 8 & FHx Complex I \\
\hline 11 & 10 & 21 & Complex II, III and IV \\
\hline 12 & 7 & 8 & Fiber Type II Predominance \\
\hline 13 & 9 & 12 & Complex III \\
\hline 14 & $<5$ & 10 & Complex III \\
\hline 15 & 12 & 16 & Neurogenic Atrophy \\
\hline 16 & $<5$ & 21 & MELAS \\
\hline
\end{tabular}

*MD = Mitochondrial Disease; FHx = family history of; ** Lowest detection limit of monitor is $5 \mathrm{ppm}$. All values below that are reported as $<5$.

\section{DISCUSSION}

Decreased serum NO has been reported in patients with $\mathrm{MD}$ and has been postulated to contribute to the pathogenesis and some of the complications of this disorder [3]. In addition, a generalized impairment of endothelial function (endothelial dysfunction) and decreased availability of the NO precursor arginine in macrophages have been reported in MD patients [3]. Since NO is produced in the respiratory system mainly by the epithelium, the endothelium, and the alveolar macrophages, the impairment in these elements of the respiratory system in patients with MD may lead to a reduction in exhaled NO production. Our pilot study demonstrates that single-breath exhaled FeNO recordings are significantly lower in patients with MD than in healthy age-matched controls.

Respiratory NO production is increased in patients with asthma and allergic rhinitis and exhaled FeNO levels can be used as a marker of lung inflammation in these populations (typically > $25 \mathrm{ppm}$ ) [13]. As many of the MD subjects in this study are highly atopic, it would be expected that FeNO levels would be elevated; however, despite their underlying atopic disease, the mitochondrial study subjects demonstrated statistically lower levels of FeNO than the non-atopic age matched controls (Tables $\mathbf{1}$ and 2). In addition, many of the subjects in the study group were taking oral L-arginine, a precursor of NO, and their FeNO levels were still decreased. 
Eleven of the MD subjects had extremely low levels of FeNO ( $\leq 7 \mathrm{ppm})$ and enzymatic deficiency in complex I was associated with these extremely low levels of NO. Interestingly, none of the MD patients with FeNO levels higher than $7 \mathrm{ppm}$ had a confirmed enzymatic deficiencies in complex I. Seven of the mitochondrial subjects had FeNO levels less than $5 \mathrm{ppm}$ as compared to one subject in the healthy control group.

The typical diseases associated with extremely low levels of FeNO is primary ciliary dyskinesia,. Levels are typically less than $5 \mathrm{ppm}$ in this population.

The diagnosis of MD is difficult in children who typically present with non-syndromic disease. Additionally, the most recognized laboratory abnormality in patients with MD is lactic acidosis and up to $70 \%$ of children with confirmed MD will have normal levels of lactic acid [19]. Hand-held FeNO measurement devices are available for use in the clinic. They are non-invasive, easy to use, and inexpensive. If our findings are corroborated by larger studies, exhaled FeNO levels could lead to a new biomarker of MD allowing for easier screening, especially in children. Measurement of exhaled FeNO could also be used as a parameter to monitor therapeutic response.

It should be noted that this study is limited by the fact that only one or two readings were performed for each subject. Current research has demonstrated low variability between Niox Mino FeNO readings [18]. However, to avoid any bias, all FeNO measurements used in the analysis were the first recorded measurement for all study subjects.

In summary, single-breath exhaled nitric oxide recordings were decreased in patients with MD. This pilot study suggests that hand-held FeNO measurements could be an attractive non-invasive biomarker of MD. Larger studies are needed to corroborate this observation.

\section{CONFLICT OF INTEREST}

The authors confirm that this article content has no conflict of interest.

\section{ACKNOWLEDGEMENTS}

We thank Joshua Samuels, MD for technical assistance in creating the tables for this article.

\section{REFERENCES}

[1] Koenig MK. Presentation and diagnosis of mitochondrial disorders in children. Pediatr Neurol 2008; 38: 305-13.
[2] Wallace DC. Mitochondrial diseases in man and mouse. Science 1999; 283: 1482-8.

[3] El-Hattab AW, Emrick LT, Craigen WJ, Scaglia F. Citrulline and arginine utility in treating nitric oxide deficiency in mitochondrial disorders. Mol Genet Metab 2012; 107(3): 247-52.

[4] Walker UA, Collins S, Byrne E. Respiratory chain encephalomyopathies: a diagnostic classification. Eur Neurol 1996; 36: 2607.

[5] Tengan $\mathrm{CH}$, Kiyomoto BH, Godinho RO, et al. The role of nitric oxide in muscle fibers with oxidative phosphorylation defects. Biochem Biophys Res Commun 2007; 359: 771-7.

[6] Koga Y, Akita Y, Nishioka J, et al. L-Arginine improves the symptoms of strokelike episodes in MELAS. Neurology 2005; 64: 710-2.

[7] Koga Y, Akita Y, Junko N, et al. Endothelial dysfunction in MELAS improved by l-arginine supplementation. Neurology 2006; 66: 1766-9.

[8] Koga Y, Akita Y, J. Nishioka J, et al. MELAS and L-arginine therapy. Mitochondrion 2007; 7: 1339.

[9] Vattemi G, Mechref Y, Marini M, et al. Increased protein nitration in mitochondrial diseases: evidence for vessel wall involvement Mol Cell Proteomics 2011; 10: 2011 (M110.002964).

[10] El-Hattab AW, Hsu JW, Emrick LT, et al. Restoration of impaired nitric oxide production in MELAS syndrome with citrulline and arginine supplementation. Mol Genet Metab 2012; 105: 607-14.

[11] Siervo M, Capuano L, Colantuoni A. Physiology and in vivo measurements of nitric oxide in man. Clin Ter 2010; 161(2): 17383.

[12] Marshall HE, Stamler JS. Exhaled Nitric Oxide (NO), NO Synthase Activity, and Regulation of Nuclear Factor (NF)- $\kappa$ B. Am J Resp Cell Mol Biol 1999; 21(3): 296-7.

[13] Manna A, Caffarelli C, Varini M, et al. Clinical application of exhaled nitric oxide measurement in pediatric lung diseases. Ital J Pediatr 2012; 38(1): 74.

[14] Girgis RE, Champion HC, Diette GB, Johns RA, Permutt S, Sylvester JT. Decreased exhaled nitric oxide in pulmonary arterial hypertension: response to bosentan therapy. Am J Respir Crit Care Med 2005; 172(3): 352-7.

[15] Paraskakis E, Zihlif N, Bush A. Nitirc oxide production in PCD: possible evidence for differential nitric oxide synthase function. Pediatr Pulmonol 2007; 42 (10): 876-80.

[16] Dweik RA, Boggs PB, Erzurum SC, et al. An official ATS clinical practice guideline: interpretation of Exhaled Nitric Oxide Levels (FENO) for clinical applications. Am J Respir Crit Care Med 2011; 184: 602-15.

[17] Keen C, Gustafsson P, Lindblad A, Wennergren G, Olin AC. Low levels of exhaled nitric oxide are associated with impaired lung function in cystic fibrosis. Pediatr Pulmonol 2010; 45(3): 241-8.

[18] Selby A, Clayton B, Grundy J, et al. Are exhaled nitric oxide measurements using the portable NIOX MINO repeatable? Respir Res 2010; 11(1): 43.

[19] Munnich A, Chretien RD, Cormier V, et al. Clinical presentation of mitochondrial disorders in childhood. J Inherit Metab Dis 1996; 19: $521-7$. 